\title{
ON THE MINIMAL FIELD OF DEFINITION OF RATIONAL MAPS: RATIONAL MAPS OF ODD SIGNATURE
}

\author{
Rubén A. Hidalgo \\ Universidad de La Frontera, Departamento de Matemática y Estadística \\ Temuco, Chile; ruben.hidalgo@ufrontera.cl
}

\begin{abstract}
The field of moduli of a rational map is an invariant under conjugation by Möbius transformations. Silverman proved that a rational map, either of even degree or equivalent to a polynomial, is definable over its field of moduli and he also provided examples of rational maps of odd degree for which such a property fails. We introduce the notion for a rational map to have odd signature and prove that this condition ensures for the field of moduli to be a field of definition. Rational maps being either of even degree or equivalent to polynomials are examples of odd signature ones.
\end{abstract}

\section{Introduction}

Two rational maps $R_{1}, R_{2} \in \mathbf{C}(z)$ are equivalent $\left(R_{1} \sim R_{2}\right)$ if one is obtained from the other by a holomorphic change of variable on the Riemann sphere $\widehat{\mathbf{C}}$, that is, if there is some Möbius transformation $T \in \mathrm{PSL}_{2}(\mathbf{C})$ so that $R_{2}=T \circ R_{1} \circ T^{-1}$. The complex dynamics of equivalent rational maps is the "same", but they may have different arithmetical properties. In this paper we are interested on (minimal) fields of definition of rational maps. A subfield $\mathbf{K}$ of $\mathbf{C}$ is called a field of definition of a rational map if there exists an equivalent rational map whose coefficients belong to $\mathbf{K}$. An interesting situation is when $\mathbf{K}=\overline{\mathbf{Q}}$ (arithmetical rational maps) as they are related to genus zero dessins d'enfants introduced by Grothendieck in his Esquisse $[4]$.

If $R$ is a rational map, a natural question is to determine if a given subfield $\mathbf{K}$ of $\mathbf{C}$ is or not a field of definition of $R$ and, in the affirmative case, to find a $\mathbf{K}$-model of it. An obstruction to the first part of the previous question can be stated in terms of certain co-boundaries. If $\operatorname{Aut}_{\mathbf{K}}(\mathbf{C})$ is the group of field automorphisms of $\mathbf{C}$, acting as the identity on $\mathbf{K}$, then a co-cycle of $R$ with respect to $\mathbf{K}$ is a map

$$
\chi: \operatorname{Aut}_{\mathbf{K}}(\mathbf{C}) \rightarrow \mathrm{PSL}_{2}(\mathbf{C}): \sigma \mapsto T_{\sigma}
$$

such that, $\forall \sigma, \tau \in \operatorname{Aut}_{\mathbf{K}}(\mathbf{C})$ the following equalities hold

$$
R^{\sigma}=T_{\sigma} \circ R \circ T_{\sigma}^{-1}, \quad T_{\sigma \tau}=T_{\tau}^{\sigma} \circ T_{\sigma},
$$

where $R^{\sigma}$ is the rational map obtained by applying $\sigma$ to the coefficients of $R$. The collection $\left\{T_{\sigma}\right\}$ is called a Weil's datum for $R$ with respect to the extension $\mathbf{C} / \mathbf{K}$. Let us observe that the existence of a co-cycle for $R$ with respect to $\mathbf{K}$ is not clear in general.

https://doi.org/10.5186/aasfm.2018.4340

2010 Mathematics Subject Classification: Primary 37P05, 37F10, 14 G05.

Key words: Rational maps, field of moduli, field of definition, Galois groups.

Partially supported by Project Fondecyt 1150003 and Anillo ACT 1415 PIA-CONICYT. 
By Weil's descent theorem [12] (see also Theorem 16.37 in page 233 in [8]), the existence of the co-cycle $\chi$ for $R$ with respect to $\mathbf{K}$ ensures the existence of a nonsingular complex projective algebraic curve of genus zero $X$, defined over $\mathbf{K}$, and a biregular isomorphism $\varphi$ : $\widehat{\mathbf{C}} \rightarrow X$, defined over $\overline{\mathbf{K}}$ (the algebraic closure of $\mathbf{K}$ inside C), such that $\varphi=\varphi^{\sigma} \circ T_{\sigma}$, for every $\sigma \in \operatorname{Aut}_{\mathbf{K}}(\mathbf{C})$. It can be seen that $\Psi=\varphi \circ R \circ \varphi^{-1}$ is also defined over $\mathbf{K}$. Now, if there is some isomorphism $L: X \rightarrow \widehat{\mathbf{C}}$, defined over $\mathbf{K}$, then $P=L \circ \varphi \in \mathrm{PSL}_{2}(\mathbf{C})$ and $P \circ R \circ P^{-1}$ is defined over $\mathbf{K}$. As a consequence of Riemann-Roch's theorem, in order to have the existence of such an $L$, we only need to check the existence of some $\mathbf{K}$-rational point in $X$. In terms of the co-cycle $\chi$, this is equivalent to being a co-boundary, that is, $\chi(\sigma)=\left(T^{\sigma}\right)^{-1} \circ T$ for a suitable Möbius transformation $T \in \mathrm{PSL}_{2}(\mathbf{C})$. Summarizing the above, the rational map $R$ can be defined over the subfield $\mathbf{K}$ if it admits a co-boundary with respect to $\mathbf{K}$.

Now, we may wonder about a "minimal field of definition" of a rational map $R$. In order to make sense of this, we have to define certain "minimal" subfield associated to $R$, this being the field of moduli $\mathcal{M}_{R}$ of $R$; the fixed field of the group $\Gamma_{R}:=\left\{\sigma \in \operatorname{Aut}_{\mathbf{Q}}(\mathbf{C}): R^{\sigma} \sim R\right\}$. Note from the definition that, if $R_{1} \sim R_{2}$, then $\Gamma_{R_{1}}=\Gamma_{R_{2}}$; so $\mathcal{M}_{R_{1}}=\mathcal{M}_{R_{2}}$. The "minimal" property of $\mathcal{M}_{R}$ is that it is contained in every field of definition of $R$. In fact, if $\mathbf{K}$ is a field of definition of $R$, then (up to equivalence) we may assume that $R$ is already defined over it; so if $\sigma \in \operatorname{Aut}_{\mathbf{K}}(\mathbf{C})$, then $R^{\sigma}=R$ and $\sigma \in \Gamma_{R}$. Following Koizumi's arguments in [7] it can be checked that $\mathcal{M}_{R}$ coincides with the intersection of all fields of definition of $R$.

Let $d$ be the degree of the rational map $R$. If $d \leq 1$, then it is not difficult to see that $\mathcal{M}_{R}$ is a field of definition of $R$. If $d \geq 2$, then the situation changes. For instance, Silverman [10] observed that, for $d \geq 3$ odd, each of the rational maps

$$
R(z)=i\left(\frac{z-1}{z+1}\right)^{d}
$$

cannot be defined over its field of moduli; which happens to be $\mathbf{Q}$. These are defined over the quadratic extension $\mathbf{Q}(i)$ and they cannot be defined over the reals (as there is no circle on the Riemann sphere invariant under $R$ ). In this example, $\mathbf{Q}(i)$ is a minimal field of definition. In the same paper, Silverman proved that every rational map, either of even degree or being equivalent to a polynomial, can be defined over its field of moduli. In [5] the author proved that every rational map can be defined over a suitable quadratic extension of its field of moduli. We do believe that if a rational map is definable over two different quadratic extensions of the same field, then it is definable over it. If this is true, then a rational map has a unique minimal field of definition.

In Section 2 we propose the definition for a rational map to be of "odd signature" and we state and prove our main result: a rational map of odd signature can be defined over its field of moduli (Theorem 1). Examples of rational maps of odd signature are those of even degree and those equivalent to polynomials. In Section 3 we provide some examples of rational maps of odd degree which have odd signature. These rational maps are given by König's rational maps $K_{P, n}$ of order $n \geq 2$, associated to a given polynomial $P$. These provide numerical algorithms to find the roots of $P$ ( $n=2$ corresponds to Newton's algorithm) $[2,3,9,11]$. We first observe that $\mathcal{M}_{K_{P, n}}$ is independent of $n$ (Proposition 1) and that $K_{P, 2}$ is definable over its field of moduli (Corollary 2). For $P$ of odd degree, $K_{P, n}$ has odd signature (generically it also has odd degree); so, by Theorem 1, it is definable over its field of moduli (Corollary 1). We conjecture that, for $P$ of even degree and $n \geq 3, K_{P, n}$ is definable over its field 
of moduli. Neverless, for the even degree situation, we provide sufficient conditions for $K_{P, n}$ to be definable over its field of moduli (Corollary 3 ).

\section{Rational maps of odd signature}

In this section we recall the definition of multipliers of periodic points of rational maps, we provide the definition of odd signature and state and prove our main result.

2.1. Fixed points and multipliers. Let $R$ be a rational map and let $p \in \widehat{\mathbf{C}}$ be a fixed point of $R$, that is, $R(p)=p$.

2.1.1. The multiplier. If $p \neq \infty$, then the multiplier of $R$ at $p$ is $\lambda_{p}=R^{\prime}(p)$. If $p=\infty$, then the multiplier $\lambda_{\infty}$ of $R$ at $\infty$ is defined as the multiplier of $1 / R(1 / z)$ at its fixed point 0 . If $\left|\lambda_{p}\right|<1$, then we say that $p$ is an attracting fixed point (when $\lambda_{p}=0$, the fixed point $p$ is called super-attracting). If $\left|\lambda_{p}\right|>1$, then $p$ is called a repelling fixed point and if $\left|\lambda_{p}\right|=1$, then it is called an indifferent fixed point.

2.1.2. The multiplicity. If $T \in \mathrm{PSL}_{2}(\mathbf{C})$, then $T(p)$ is a fixed point of $S=$ $T \circ R \circ T^{-1}$ with the same multiplier. If $T(p)=0$, then $S(z)=z^{m_{p}} Q(z)$, where $Q(0) \neq 0$ and $m_{p} \geq 1$ is some integer; called the multiplicity of $p$ (this does not depend on the choice of $T$ ). If $m_{p}=1$, then $\lambda_{p}=Q(0) \neq 0$ and, if $m_{p} \geq 2$, then $\lambda_{p}=0$ (i.e., $p$ is a super-attracting fixed point).

Remark 1. Let $\left\{\lambda_{1}, \ldots, \lambda_{r}\right\}$ be the collection of all non-zero multipliers of fixed points of $R$ and let $p$ be a super-attracting fixed point of multiplicity $m \geq 2$. If $\sigma \in \Gamma_{R}$, then as $R^{\sigma} \sim R$, one has that $\left\{\sigma\left(\lambda_{1}\right), \ldots, \sigma\left(\lambda_{r}\right)\right\}=\left\{\lambda_{1}, \ldots, \lambda_{r}\right\}$ and $\sigma(p)$ is a super-attracting fixed point of $R^{\sigma}$ with multiplicity $m$.

2.2. Rational maps of odd signature. We say that the rational map $R$ has odd signature if there is some integer $k \geq 1$ so that one of the following properties holds (see also Remark 1).

(OS1) There exists an odd number of fixed points of $R^{k}$ (the $k$-iterate of $R$ ), each one being not super-attracting, so that the set formed by their multipliers is $\Gamma_{R}$-invariant, and every another fixed point of $R^{k}$ has multiplier different from these.

(OS2) There is some integer $m \geq 2$ so that the number of supper-attracting fixed points of $R^{k}$ with multiplicity equal to $m$ is odd.

The rational map $R$ has simple odd signature if in the above definition we may assume $k=1$. Next, we state our main result.

Theorem 1. A rational map of odd signature is definable over its field of moduli.

Remark 2. (Silverman's result) Let us observe that if $R^{k}$ has an odd number of fixed points, then $R$ has odd signature (see Remark 1). If $R$ has even degree, then it has an odd number of fixed points (counting multiplicities), so it has simple odd signature. If $R$ is a polynomial of degree $d \geq 2$, then $\infty$ is a super-attracting fixed point with multiplicity $d$. Either this is the only super-attracting fixed point (so it has simple odd signature) or $R$ is conjugated to $z \mapsto z^{d}$ (which is defined over its field of moduli). In this way, Silverman's result [10] also follows from Theorem 1.

2.3. Proof of Theorem 1. Let $R$ be a rational map of degree $d \geq 2$ of odd signature. So, there exists a collection of fixed points of $R^{k}$, say $z_{1}, \ldots, z_{n}$, where $n \geq 1$ is odd, for which one of the conditions (OS1) or (OS2) holds. The proof of the following fact will be provided in Section 2.5. 
Lemma 1. $R$ admits a Weil's datum $\left\{T_{\sigma}\right\}_{\sigma \in \Gamma_{R}}$.

As observed in the introduction, both Lemma 1 and Weil's descent theorem [12] ensure the existence of a genus zero smooth complex curve $X$, defined over $\mathbf{K}$, and an isomorphism $\varphi: \widehat{\mathbf{C}} \rightarrow X$ so that, for every $\sigma \in \Gamma_{R}$ it holds that $\varphi=\varphi^{\sigma} \circ T_{\sigma}$. If $\Psi=\varphi \circ R \circ \varphi^{-1}: X \rightarrow X$, then for $\sigma \in \Gamma_{R}$

$$
\Psi^{\sigma}=\varphi^{\sigma} \circ R^{\sigma} \circ\left(\varphi^{\sigma}\right)^{-1}=\varphi^{\sigma} \circ T_{\sigma} \circ R \circ T_{\sigma}^{-1} \circ\left(\varphi^{\sigma}\right)^{-1}=\varphi \circ R \circ \varphi^{-1}=\Psi .
$$

Lemma 2. The divisor $D=\left[\varphi\left(z_{1}\right)\right]+\cdots+\left[\varphi\left(z_{n}\right)\right]$ in $X$ is $\mathcal{M}_{R^{-r a t i o n a l}}$

Proof. Let us first assume we are in condition (OS1) and let $\lambda_{j} \neq 0$ be the multiplier of $z_{j}$, for $j=1, \ldots, n$. So, if $\sigma \in \Gamma_{R}$, then $\left\{\lambda_{1}, \ldots, \lambda_{n}\right\}=\left\{\sigma\left(\lambda_{1}\right), \ldots, \sigma\left(\lambda_{n}\right)\right\}$. If $z$ is a fixed point of $R^{k}$ with multiplier $\lambda$, then $T_{\sigma}(z)$ is a fixed point of $\left(R^{k}\right)^{\sigma}$ with same multiplier $\lambda$. It follows that $\left\{T_{\sigma}\left(z_{1}\right), \ldots, T_{\sigma}\left(z_{n}\right)\right\}$ are the fixed points of $\left(R^{k}\right)^{\sigma}$ with multipliers in the set $\left\{\lambda_{1}, \ldots, \lambda_{n}\right\}$. Also, as $\sigma\left(z_{j}\right)$ is a fixed point of $\left(R^{k}\right)^{\sigma}$ with multiplier $\sigma\left(\lambda_{j}\right)$, the set $\left\{\sigma\left(z_{1}\right), \ldots, \sigma\left(z_{n}\right)\right\}$ also consists of all the fixed points of $\left(R^{k}\right)^{\sigma}$ with multipliers in the set $\left\{\lambda_{1}, \ldots, \lambda_{n}\right\}$. It follows that $\left\{T_{\sigma}\left(z_{1}\right), \ldots, T_{\sigma}\left(z_{n}\right)\right\}=$ $\left\{\sigma\left(z_{1}\right), \ldots, \sigma\left(z_{n}\right)\right\}$ and, in particular, $\sigma$ induces a permutation $\theta_{\sigma} \in \mathfrak{S}_{n}$ so that $T_{\sigma}\left(z_{j}\right)=\sigma\left(z_{\theta_{\sigma}(j)}\right)$. In this way,

$$
\varphi\left(z_{j}\right)=\varphi^{\sigma}\left(T_{\sigma}\left(z_{j}\right)\right)=\varphi^{\sigma}\left(\sigma\left(z_{\theta_{\sigma}(j)}\right)\right)=\sigma\left(\varphi\left(z_{\theta_{\sigma}(j)}\right)\right) .
$$

The above ensures that the set $\left\{\varphi\left(z_{1}\right), \ldots, \varphi\left(z_{n}\right)\right\}$ is $\Gamma_{R}$-invariant and the result follows in this case. In the case (OS2), that is, the collection $\left\{z_{1}, \ldots, z_{n}\right\}$ are the supper-attracting fixed points with the same multiplicity, the argument is similar as above as the multiplicity will not change under $T_{\sigma}$ nor under $\sigma$.

By Lemma 3 below, there is an isomorphism $\eta: X \rightarrow \widehat{\mathbf{C}}$ defined over $\mathcal{M}_{R}$; so the rational map $S=\eta \circ \Psi \circ \eta^{-1}$ satisfies that $S^{\sigma}=S$, for every $\sigma \in \Gamma_{R}$. It follows that $S$ is defined over $\mathcal{M}_{R}$. In fact, let us write $S(z)=U(z) / V(z)$, where $U$ and $V$ are relatively prime polynomials, and the leading coefficient of $V$ is equal to 1 . The condition $S^{\sigma}=S$, for $\sigma \in \Gamma_{R}$, ensures the existence of a non-zero complex number $a(\sigma)$ such that $U^{\sigma}=a(\sigma) U$ and $V^{\sigma}=a(\sigma) V$. Our normalization asserts that $a(\sigma)=1$, that is, $U$ and $V$ (so $S$ ) are defined over $\mathcal{M}_{R}$ as claimed. Now, $T=\eta \circ \varphi: \widehat{\mathbf{C}} \rightarrow \widehat{\mathbf{C}}$ is a Möbius transformation (i.e., an element of $\mathrm{PSL}_{2}(\mathbf{C})$ ) with $T \circ R \circ T^{-1}=S$; we have proved the theorem.

\subsection{An auxiliary Lemma.}

Lemma 3. [6] Let $B$ be a curve of genus zero defined over a subfield $\mathbf{K}$ of $\mathbf{C}$. If there is a $\mathbf{K}$-rational divisor $D$ of odd degree in $B$, then there exists an isomorphism $\eta: B \rightarrow \widehat{\mathbf{C}}$ defined over $\mathbf{K}$ and, in particular, $B$ has (infinitely many) K-rational points.

Proof. Let us assume the degree of $D$ is $2 m+1$, for a suitable non-negative integer $m$. As $B$ has genus zero and is defined over $\mathbf{K}$, there is a canonical divisor $K_{B}$ of $B$, defined over $\mathbf{K}$. As $K_{B}$ has degree -2 , the $\mathbf{K}$-rational divisor $U=D+m K_{B}$ has degree 1. By Riemann-Roch's theorem, the Riemann-Roch's space

$$
\mathcal{L}(U)=\{f: B \rightarrow \widehat{\mathbf{C}}:(f)+U \geq 0\} \cup\{0\}
$$

has dimension 2. Since $U$ is $\mathbf{K}$-rational, we may find some $f \in \mathcal{L}(U)-\{0\}$ which is defined over $\mathbf{K}$. So the $\mathbf{K}$-rational divisor $U+(f)$ is an effective divisor of degree 1 , that is, a K-rational point $q$ on $B$. As the dimension of the Riemann-Roch's space $\mathcal{L}(q)$ is 2 , it follows that there is an isomorphism $\eta: B \rightarrow \widehat{\mathbf{C}}$ defined over $\mathbf{K}$. As $\widehat{\mathbf{C}}$ 
has infinitely many $\mathbf{K}$-rational points, and $\eta$ is defined over $\mathbf{K}$, the same holds with $B$.

2.5. The proof of Lemma 1. Let $\operatorname{Aut}(R)$ be the group of automorphisms of $R$, that is, the group of Möbius transformations $T \in \mathrm{PSL}_{2}(\mathbf{C})$ satisfying the equality $T \circ R=R \circ T$. As the degree of $R$ is at least two, this group is known to be finite (a Möbius transformation is uniquely determined by its action on three different points). Since the finite groups of $\mathrm{PSL}_{2}(\mathbf{C})$ are either (i) the trivial group, (ii) cyclic groups, (iii) dihedral groups, (iv) the alternating group $\mathcal{A}_{4}$, (v) the alternating group $\mathcal{A}_{5}$ and (vi) the symmetric group $\mathfrak{S}_{4}$ (see, for instance, [1]), the group $\operatorname{Aut}(R)$ is either one of the above.

2.5.1. Assume $\operatorname{Aut}(R)$ is the trivial group. For each $\sigma \in \Gamma_{R}$ there is some $T_{\sigma} \in \mathrm{PSL}_{2}(\mathbf{C})$ so that $R^{\sigma}=T_{\sigma} \circ R \circ T_{\sigma}^{-1}$. As $\operatorname{Aut}(R)$ is trivial, $T_{\sigma}$ is unique with such property. Clearly, $\left\{T_{\sigma}\right\}_{\sigma \in \Gamma_{R}}$ is a Weil's datum for $R$ with respect to the extension $\mathrm{C} / \mathcal{M}_{R}$.

2.5.2. Assume $\operatorname{Aut}(R)$ is either dihedral, alternating or the symmetric group. In this case, there is a branched regular cover $\pi: \widehat{\mathbf{C}} \rightarrow \widehat{\mathbf{C}}$ with $\operatorname{Aut}(R)$ as deck group and branch values being the points $\infty, 0$ and 1 . Next, for each $\sigma \in \Gamma_{R}$ we take some Möbius transformation $L_{\sigma} \in \mathrm{PSL}_{2}(\mathbf{C})$ so that $R^{\sigma}=L_{\sigma} \circ R \circ L_{\sigma}^{-1}$. As $L_{\sigma}$ conjugates $\operatorname{Aut}(R)$ onto $\operatorname{Aut}\left(R^{\sigma}\right)$, then there is a Möbius transformation $N_{\sigma}$ so that $N_{\sigma} \circ \pi=\pi^{\sigma} \circ L_{\sigma}$. The map $N_{\sigma}$ only depends on $\sigma$ and not on the choice for $L_{\sigma}$. In fact, if $L \in \mathrm{PSL}_{2}(\mathbf{C})$ satisfies that $R^{\sigma}=L \circ R \circ L^{-1}$, then $H=L^{-1} \circ L_{\sigma} \in \operatorname{Aut}(R)$. In this way, $\pi^{\sigma} \circ L=\pi^{\sigma} \circ L_{\sigma} \circ H^{-1}=N_{\sigma} \circ \pi \circ H^{-1}=N_{\sigma} \circ \pi$. The uniqueness property on the maps $N_{\sigma}$ ensures that $\left\{N_{\sigma}\right\}_{\sigma \in \Gamma_{R}}$ satisfies, for each $\tau, \sigma \in \Gamma_{R}$, the equality $N_{\sigma \tau}=N_{\tau}^{\sigma} \circ N_{\sigma}$. It now follows from Weil's descent theorem [12] the existence of a smooth curve $B$, defined over $\mathcal{M}_{R}$, and an isomorphism $\tau: \widehat{\mathbf{C}} \rightarrow B$ so that $\tau=\tau^{\sigma} \circ N_{\sigma}$, for every $\sigma \in \Gamma_{R}$. As the divisor $D=[\tau(\infty)]+[\tau(0)]+[\tau(1)]$ is

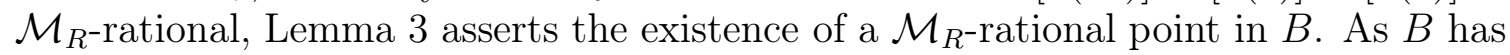
genus zero, it follows that $B$ has infinitely many $\mathcal{M}_{R}$-rational points. Take one of these points, say $q$, outside the branch values of $\tau \circ \pi$. Choose a point $p \in \widehat{\mathbf{C}}$ so that $\tau \circ \pi(p)=q$; so it is not fixed by any non-trivial element of $\operatorname{Aut}(R)$. If $\sigma \in \Gamma_{R}$, then

$$
\begin{aligned}
(\tau \circ \pi)^{\sigma}(\sigma(p)) & =\sigma(\tau(\pi(p)))=\sigma(q)=q=\tau \circ \pi(p)=\tau^{\sigma} \circ N_{\sigma} \circ \pi(p) \\
& =\tau^{\sigma} \circ \pi^{\sigma}\left(L_{\sigma}(p)\right)=(\tau \circ \pi)^{\sigma}\left(L_{\sigma}(p)\right),
\end{aligned}
$$

that is, there is some $M_{\sigma} \in \operatorname{Aut}\left(R^{\sigma}\right)$ so that $\left(M_{\sigma} \circ L_{\sigma}\right)(p)=\sigma(p)$. We claim that $M_{\sigma}$ is unique. In fact, if there is some $D_{\sigma} \in \operatorname{Aut}\left(R^{\sigma}\right)$ satisfying that $\left(D_{\sigma} \circ L_{\sigma}\right)(p)=\sigma(p)$, then $D_{\sigma}^{-1} \circ M_{\sigma}$ fixes $L_{\sigma}(p)$. Since we know that $p$ is not fixed by any non-trivial element of $\operatorname{Aut}(R)$, the same holds for $L_{\sigma}(p)$ and $\operatorname{Aut}\left(R^{\sigma}\right)$. It follows that $D_{\sigma}^{-1} \circ M_{\sigma}$ is the identity, i.e., $D_{\sigma}=M_{\sigma}$. Now, the collection $\left\{T_{\sigma}=M_{\sigma} \circ L_{\sigma}\right\}_{\sigma \in \Gamma_{R}}$ turns out to be a Weil's datum for $R$ with respect to the extension $\mathbf{C} / \mathcal{M}_{R}$.

2.5.3. Assume $\operatorname{Aut}(R)$ is a non-trivial cyclic group of order $b \geq 2$, say generated by the transformation $E \in \mathrm{PSL}_{2}(\mathbf{C})$. Let us consider a branched regular cover $\pi: \widehat{\mathbf{C}} \rightarrow \widehat{\mathbf{C}}$, with $\operatorname{Aut}(R)$ as deck group, whose branch values are the points $\infty$ and 0. Proceeding as in 2.5.2, we obtain a collection $\left\{N_{\sigma}\right\}_{\sigma \in \Gamma_{R}}$ (such that for each $\tau, \sigma \in \Gamma_{R}$, the equality $N_{\sigma \tau}=N_{\tau}^{\sigma} \circ N_{\sigma}$ holds), a smooth curve $B$, defined over $\mathcal{M}_{R}$, and an isomorphism $\tau: \widehat{\mathbf{C}} \rightarrow B$ so that $\tau=\tau^{\sigma} \circ N_{\sigma}$, for every $\sigma \in \Gamma_{R}$. The elliptic transformation $E$ must keep the set $F=\left\{z_{1}, \ldots, z_{n}\right\}$ invariant. Assume that $b$ is even. In this case, exactly one of the points in $F$ should be fixed point of $E$. We may 
assume, without lost of generality, that this point is $z_{1}$ and that $\pi\left(z_{1}\right)=\infty$. Now we may proceed similarly as done in 2.5 .2 by considering the $\mathcal{M}_{R^{-} \text {rational divisor }}$ $D=[\tau(\infty)]$ to obtain the desired Weil's datum. Now, assume that $b$ is odd. In this case, as $n$ is odd, it follows that the projection under $\pi$ of $F$ is a set of odd cardinality, say given by the points $a_{1}, \ldots, a_{t}$ ( $t$ odd). We now proceed as in 2.5.2 by considering the $\mathcal{M}_{R^{-}}$rational divisor $D=\left[\tau\left(a_{1}\right)\right]+\cdots+\left[\tau\left(a_{t}\right)\right]$ to obtain the desired Weil's datum.

Example 1. Silverman's rational map $R(z)=i\left(\frac{z-1}{z+1}\right)^{3}$ has exactly 4 fixed points, these being $z_{1}=-3.04757 \ldots+i 2.12615 \ldots, z_{2}=-0.28211 \ldots-i 1.58405 \ldots$, $z_{3}=0.10897 \ldots+i 0.61188 \ldots$ and $z_{4}=0.22070 \ldots-i 0.15397 \ldots$, with corresponding multipliers $\bar{\lambda}_{z_{4}}=\lambda_{z_{1}}=-1.2859 \ldots-i 1.0372 \ldots$ and $\bar{\lambda}_{z_{3}}=\lambda_{z_{2}}=-0.2141 \ldots+i 2.7154 \ldots$ If $\sigma \in \Gamma_{R}$, then $\sigma(i)= \pm i$; so the $\Gamma_{R^{-}}$equivalent sets of multipliers are $\left\{\lambda_{z_{1}}, \lambda_{z_{4}}\right\}$, $\left\{\lambda_{z_{2}}, \lambda_{z_{3}}\right\}$ and $\left\{\lambda_{z_{1}}, \lambda_{z_{4}}, \lambda_{z_{2}}, \lambda_{z_{3}}\right\}$. In particular, $R$ does not have simple odd signature. As a consequence of Theorem 1, and the fact that it cannot be defined over its field of moduli [10], it neither has odd signature.

\section{Examples: König's rational maps}

If $P(z) \in \mathbf{C}[z]$ is a polynomial of degree $d \geq 2$ and $n \geq 2$ is an integer, then its König's rational map of order $n$ is defined as

$$
K_{P, n}(z)=z+(n-1) \frac{(1 / P)^{(n-2)}(z)}{(1 / P)^{(n-1)}(z)},
$$

where $R^{(k)}$ denotes the $k$-derivative of $R$ [9]; it has degree at most $(n-1)(d-1)+1$. A characterization and study of the dynamics of König's rational maps has been given in [2]. The rational map $K_{P, 2}(z)=z-P(z) / P^{\prime}(z)$ is Newton's rational map associated to $P$ and $K_{P, 3}$ provides the so-called Halley's method to find roots of $P$ [11] (choose a point $z_{0} \in \mathbf{C}$ and define $z_{1} \in \widehat{\mathbf{C}}$ the unique zero of the Möbius transformation that better "osculate" $P$ in the point $z_{0}$ ). The fixed points of $K_{P, n}$ are given by:

(1) the zeroes of $P$; these being the (super) attracting fixed points of $K_{P, n}$;

(2) the zeroes of $(1 / P)^{(n-2)}$ and the point $\infty$; these being the repelling fixed points of $K_{P, n}$.

Remark 3. The field of moduli of a polynomial $P$ and the field of moduli of its König's rational map $K_{P, n}$ may not coincide. For instance, if $P_{c}(z)=z^{2}+c$, where $c \in \mathbf{C}-\{0\}$, then $K_{P_{c}, n}$ is conjugated to $R(z)=z^{n}$ (see Section 2.2. in [2]); so it is definable over its field of moduli $\mathbf{Q}$. On the other hand, $\mathcal{M}_{P_{c}}=\mathbf{Q}(c)$.

3.1. Invariance of the field of moduli of König's rational maps. It is not difficult to see that $K_{P, n}=K_{Q, n}$ if and only if $Q=\mu P$, where $\mu \neq 0$. In particular, if we assume our polynomials to be monic, then the above ensures $\mu=1$. On the other hand, Lemma 9 in [2] states that $K_{P, n} \sim K_{Q, n}$ if and only if there exist $a, b, \mu \in \mathbf{C}$ with $a \mu \neq 0$ so that $Q(z)=\mu P(a z+b)$. This permits to prove the following fact.

Proposition 1. If $n \geq 2$, then $\mathcal{M}_{K_{P, n}}$ is independent of $n$.

Proof. Since $K_{P, n}^{\sigma}=K_{P^{\sigma}, n}$, one has that $\sigma \in \Gamma_{K_{P, n}}$ if and only if $K_{P^{\sigma}, n} \sim K_{P, n}$, which is equivalent to $P^{\sigma}(z)=\mu P(a z+b)$, for suitable $a, b, \mu \in \mathbf{C}$ with $a \mu \neq 0$. But the last condition is independent of $n$, so $\Gamma_{K_{P, n}}=\Gamma_{K_{P, 2}}$. It follows that the field of moduli of $K_{P, n}$ is equal to the field of moduli of $K_{P, 2}$. 
3.2. König's rational maps of odd degree polynomials. As the (super) attracting fixed points of $K_{P, n}$ are given by the zeroes of $P$, if the degree of $P$ is odd, then $K_{P, n}$ will be of odd signature; so, by Theorem 1, we have the following.

Corollary 1. If $P$ is a polynomial of odd degree, then $K_{P, n}$ is definable over its field of moduli, for every integer $n \geq 2$.

Let us note that $K_{P, n}$ will have (generally) odd degree $(n-1)(d-1)+1$, so the above result is not a consequence of Silverman's results in [10].

3.3. An example. Let us consider the degree three polynomial

$$
P(z)=(z-1)(z-a)(z+a+1), \quad a \in \mathbf{C}-\{-2,-1 / 2,1\} .
$$

By Corollary $1, K_{P, n}$ is definable over its field of moduli (which is, by Proposition 1 , independent of $n \geq 2$ ). If $a \in \mathbf{Q}$, then $K_{P, n}$ is already defined over $\mathbf{Q}$. If $a=i$, then the field of moduli of $K_{P, n}$ is again $\mathbf{Q}$, and for $T(z)=(1-i) z$ one has that $T \circ K_{P, n} \circ T^{-1}(z)$ is defined over $\mathbf{Q}$.

\subsection{Newton's rational map.}

Corollary 2. If $P$ is a polynomial of degree $d \geq 2$, then Newton's rational map $K_{P, 2}$ is definable over its field of moduli.

Proof. Let $P(z) \in \mathbf{C}[z]$ be a polynomial of degree $d \geq 2$. If $d$ is odd, then the result follows from Corollary 1 . We now assume $d \geq 2$ even. If $P(z)=a(z / d+b)^{d}$, then $K_{P, 2}(z)=(d-1) z / d-b$ has degree 1 , so it is definable over its field of moduli. If $P(z) \neq a(z / d+b)^{d}$, then $K_{P, 2}$ is a rational map of degree $d$. As $d$ is even, $K_{P, 2}$ is definable over its field of moduli by Silverman's results [10].

3.5. König's rational maps of even degree polynomials. Proposition 3 in [2] states that for a generic polynomial $P$ of degree $d$ its König's rational map $K_{P, n}$ has $(n-2)(d-1)$ repelling fixed points in $\mathbf{C}$ and their multipliers are all equal to $n$. So, if $d \geq 3$, then $1+(n-1) /(d-1) \neq n$ and, in particular, $\infty$ is the unique repelling fixed point with multiplier $1+(n-1) /(d-1)$. So Theorem 1 ensures that, for generic $P$ of even degree $d \geq 2$, the rational map $K_{P, n}$ can be defined over its field of moduli.

Conjecture 1. If $P$ is a polynomial of even degree $d \geq 2$ and $n \geq 3$ is an integer, then König's rational map $K_{P, n}$ can be defined over its field of moduli.

Remark 4. In Remark in page 10 of [2] it was noticed that, for each polynomial $P(z)=\mu(z-1) z^{d}$ and each integer $n \geq 2$, there is a polynomial $Q$, of degree $n$, so that $K_{P, n}=Q \circ T$, where $T(z)=1 /(z-1)$. Since polynomials are definable over their field of moduli (and $T$ is already defined over $\mathbf{Q}$ ), we expect the same to be true for $K_{P, n}$ (recall that we know this is the case if $d$ is odd).

The fixed point $\infty$ of $K_{P, n}$ has multiplier $1+(n-1) /(d-1)$ and, if $p \in \mathbf{C}$ is a zero of $(1 / P)^{(n-2)}$ with multiplicity $m$, then its multiplier for $K_{P, n}$ is $1+(n-1) / m$; see Lemma 3 in $[3,2]$. As these multipliers are rational numbers, so they are fixed points of the elements of $\Gamma_{K_{P, n}}$, to check if $K_{P, n}$ has simple odd signature is easy; just count how many fixed points of $K_{P, n}$ have the same multiplier. This is summarized in the following.

Lemma 4. $K_{P, n}$ has simple odd signature (so it is definable over its field of moduli) if and only if it has an odd number of fixed points with a given multiplier. 
Corollary 3. Let $P$ be a polynomial of even degree $d \geq 2$ and let $n \geq 3$ be an integer. Then König's rational map $K_{P, n}$ can be defined over its field of moduli if each zero of $(1 / P)^{(n-2)}$ has multiplicity different from $d-1$.

Proof. In this case, the point $\infty$ is the only repelling fixed point of $K_{P, n}$ with multiplier equal to $1+(n-1) /(d-1)$. Now the result follows from Lemma 4 and Theorem 1.

Acknowledgments. I am grateful to the referee whose suggestions helped to improve the presentation of the paper.

\section{References}

[1] Beardon, A. F.: The geometry of discrete groups. Corrected reprint of the 1983 original. Grad. Texts in Math. 91, Springer-Verlag, New York, 1995.

[2] Buff, X., and Ch. Henriksen: On König's root-finding algorithms. - Nonlinearity 16:3, 2003, 989-1015.

[3] Doyle, P., and C. McMullen: Solving the quintic by iteration. - Acta Math. 163:3-4, 1989, $151-180$.

[4] Grothendieck, A.: Esquisse d'un programme (1984). - In: Geometric Galois actions, L. Schneps and P. Lochak eds., London Math. Soc. Lect. Notes Ser. 242, Cambridge Univ. Press, Cambridge, 1997, 5-47.

[5] Hidalgo, R. A.: A simple remark on the field of moduli of rational maps. - Quart. J. Math. $65,2014,627-635$.

[6] Huggins, B.: Fields of moduli of hyperelliptic curves. - Math. Res. Lett. 14:2, 2007, 249-262.

[7] Koizumi, S.: Fields of moduli for polarized Abelian varieties and for curves. - Nagoya Math. J. 48, 1972, 37-55.

[8] Milne, J.: Algebraic geometry. - http://www.jmilne.org/math/CourseNotes/AG.pdf.

[9] SchröDER, E.: Über unendlich viele Algoritmen zur Auflösung der Gleichungen. - Math. Ann. $2,1870,317-365$.

[10] Silverman, J. H.: The field of definition for dynamical systems on $\mathbf{P}^{1}$. - Compos. Math. 98:3, 1995, 269-304.

[11] Scavo, T. R., and J. B. Thoo: On the geometry of Halley's method. - Amer. Math. Monthly 102:5, 1995, 417-426.

[12] WeIL, A.: The field of definition of a variety. - Amer. J. Math. 78, 1956, 509-524.

Received 21 August 2015• Revised received 5 July 2017 • Accepted 22 December 2017 